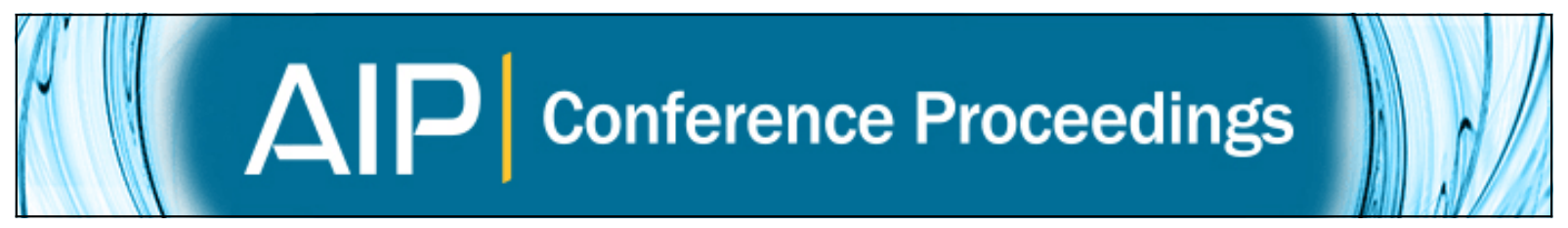

Prediction of inertial effects due to bone conduction in a 2D box model of the cochlea

Alice A. Halpin, Stephen J. Elliott, and Guangjian Ni

Citation: AIP Conference Proceedings 1703, 060015 (2015); doi: 10.1063/1.4939370

View online: http://dx.doi.org/10.1063/1.4939370

View Table of Contents: http://scitation.aip.org/content/aip/proceeding/aipcp/1703?ver=pdfcov

Published by the AIP Publishing

Articles you may be interested in

A tapered box model of the cochlea

AIP Conf. Proc. 1703, 070014 (2015); 10.1063/1.4939388

Finite element cochlea box model - Mechanical and electrical analysis of the cochlea

AIP Conf. Proc. 1703, 070012 (2015); 10.1063/1.4939386

Cochlear boundary motion during bone conduction stimulation: Implications for inertial and compressional excitation of the cochlea

AIP Conf. Proc. 1703, 060005 (2015); 10.1063/1.4939360

Pressures in the human cochlea during bone conduction

AIP Conf. Proc. 1703, 060004 (2015); 10.1063/1.4939359

Wave Motion in the Cochlea Caused by Bone Conduction

J. Acoust. Soc. Am. 25, 986 (1953); 10.1121/1.1907231 


\title{
Prediction of Inertial Effects due to Bone Conduction in a 2D Box Model of the Cochlea
}

\author{
Alice A. Halpin, Stephen J. Elliott and Guangjian Ni \\ Institute of Sound and Vibration Research, University of Southampton, Southampton, UK
}

\begin{abstract}
A 2D box model of the cochlea has been used to predict the basilar membrane, BM, velocity and the fluid flow caused by two components of bone conduction: due to inertia of the middle ear and due to inertia of the cochlear fluids. A finite difference approach has been used with asymmetric fluid chambers, that enables an investigation of the effect of varying window stiffness, due to otosclerosis for example. The BM is represented as a series of locally reacting single degree of freedom systems, with graded stiffness along the cochlea to represent the distribution of natural frequencies and with a damping representative of the passive cochlea. The velocity distributions along the passive BM are similar for harmonic excitation via the middle ear inertia or via the fluid inertia, but the variation of the BM velocity magnitude with excitation frequency is different in the two cases. Excitation via the middle ear is suppressed if the oval window is assumed to be blocked, but the excitation via the cochlear fluids is still possible. By assuming a combined excitation due to both middle ear and fluid excitation, the difference between the overall response can be calculated with a flexible and a blocked oval window, which gives a reasonable prediction of Carhart's notch.
\end{abstract}

\section{INTRODUCTION}

Bone conduction, $\mathrm{BC}$, hearing is the transmission of vibrations through the skull bone to the inner ear, stimulating the cochlea and ultimately the basilar membrane, BM, to produce a hearing sensation. The concept of hearing through $\mathrm{BC}$ has been recognised and researched for centuries. BC hearing has been useful for many years in a clinical setting and more recently commercially. There are multiple mechanisms in which vibrations of the skull can cause a hearing sensation. Stenfelt [7] have classified the mechanisms into five pathways, which are:

1. Sound pressure in the ear-canal and the occlusion effect

2. Compression and expansion of the cochlea walls

3. Transmission of pressure from the cerebrospinal fluid

4. Inertia of the middle ear ossicles

5. Inertia of the cochlear fluid

The first of these mechanism is due to the vibrations of the skull causing compression and expansion of the ear canal that produces a sound pressure. The sound wave can then cause a hearing sensation by the same process as air conduction hearing. This mechanism only becomes a significant pathway if the ear canal is occluded [8]. At high frequencies the cochlear walls compress and expand, deforming the space inside cochlea causing a travelling wave along the BM and hence a hearing sensation [9]. Vibrations of the skull are thought to cause pressure waves in the cerebrospinal fluid which are believed to be transmitted to the cochlea through fluid channels such as the cochlear aqueduct, vestibular aqueduct and blood vessels [6]. The last two mechanisms are due to inertia effects. Vibrations of the skull can produce a relative motion between the middle ear ossicles and the surrounding bone causing the stapes to oscillate and stimulate the cochlea. Inertial forces acting on the cochlear fluids can cause motion of the BM. The overall effect of $\mathrm{BC}$ is due to a vectorial combination of these pathways, where the significance of the contribution of each pathway changes with excitation frequency.

In reality, all these pathways are acting simultaneously and so it is impossible to distinguish between them. Due to the complicated structure of the biological system it is impossible to experimentally isolate each pathway in vivo. Here, a 2D finite difference box model has been used to separately estimated the BM response for two BC pathways, the middle ear inertia and the fluid inertia. Modelling the two inertial BC pathways using the same cochlea model enabled a comparison of the BM response, per skull displacement, between each pathway. The magnitude of the maximum BM displacements, calculated for both BC pathways, were compared over a range of frequencies. It was then possible to predict which component is more significant to $\mathrm{BC}$ hearing over the frequencies range investigated. By summing the 
complex BM displacements for each pathway together, a total BM response to $\mathrm{BC}$ hearing was predicted. By altering the parameters of the model an otosclerosis diseased ear was modelled and the effect on the BC response calculated.

\section{METHOD}

A 2D box model of the passive cochlea, solved using a finite difference method, is presented here. The cochlea is assumed to be uncoiled and so modelled as two rectangular fluid filled chambers separated by an elastic membrane of varying stiffness, the BM. The cochlear windows are positioned at the base of the cochlea on the walls perpendicular to the BM of each chamber. The box model was split into 294 elements, $N=295$ nodes, in the $x$-direction and 21 elements, $M=22$ nodes, in the $z$-direction with the BM having a thickness of two elements and located so that the cochlear is split in the ratio of $1: 2$, as shown in Fig. 1.

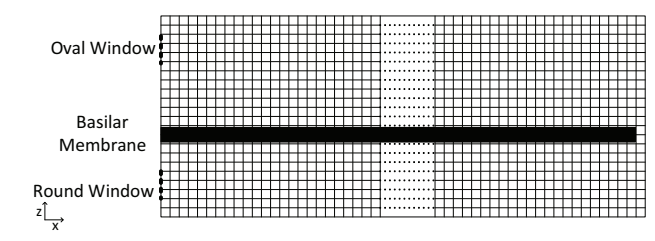

FIGURE 1. The $2 \mathrm{D}$ box model and the division of the two fluid chambers into $\mathrm{N}$ nodes in the $\mathrm{X}$-direction and $\mathrm{M}$ nodes in the $\mathrm{Z}$-direction.

The fluid in the chambers is assumed to be incompressible, inviscid and only flow in the $x$ and $z$ directions and so satisfies the Laplace equation,

$$
\frac{\partial^{2}}{\partial x^{2}} p(x, z)+\frac{\partial^{2}}{\partial y^{2}} p(x, z)=0 .
$$

The boundary conditions define the acceleration of the fluid at certain locations by the pressure gradient in either the $x$ or $z$ direction,

$$
\frac{\partial p(x, z)}{\partial z}=-\rho a_{z}, \quad \frac{\partial p(x, z)}{\partial x}=-\rho a_{x} .
$$

The BM extends from the base of the cochlea and ends at the Helicotrema, which is located at the apex. It is modelled as a series of locally reacting single degree of freedom systems shown in Fig. 2, where each system is one element wide and two elements thick. The base of the system is assumed to move with the velocity of the lower cochlear wall, $v_{w}$. The stiffness, $k_{B M}(x)$ and damping $c_{B M}(x)$ vary along its length as,

$$
k_{B M}(x)=\omega_{B}^{2} m_{o} e^{\frac{-2 x}{l}}, \quad c_{B M}(x)=\frac{\sqrt{k_{B M} m_{o}}}{Q_{o}}
$$

representing the distribution of natural frequencies along the cochlea and with a damping representative of the passive cochlea. $\omega_{B}$ is the natural frequency at the base of the cochlea, $20 \mathrm{kHz}$, and $Q_{o}$ is a constant estimated to equal 2.5 in a passive cochlea [3]. $l$, is the characteristic length, which here it has been set to equal $7 \times 10^{-3} \mathrm{~m}$. The mass per unit area of each section of the $\mathrm{BM}$ is assumed to be constant, $m_{o}=2 \rho \Delta z \mathrm{kgm}^{-2}$, where $\rho$ is the density of water, $\Delta z$, the thickness of one element and the factor of 2 due to the BM being two elements thick. It is assumed that there is no longitudinal coupling along the BM and hence the acceleration of the $\mathrm{BM}$, due to both the fluid pressure difference, $p_{d}(x)$, and the wall velocity, $v_{w}$, is

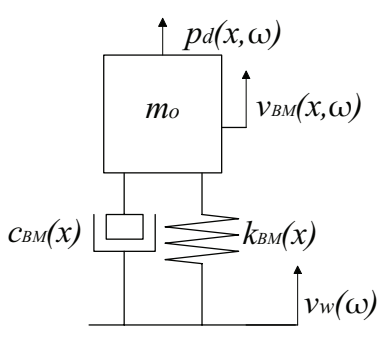

FIGURE 2. Diagram of one locally reacting single degree of freedom system that is part of a series representing the BM.

location $(n \Delta x, m \Delta z)$, which is written for conveniences as $[n, m]$, can be expressed as,

$$
\frac{p[n+1, m]-2 p[n, m]+p[n-1, m]}{\Delta x^{2}}+\frac{p[n, m+1]-2 p[n, m]+p[n, m-1]}{\Delta z^{2}}=0,
$$


where $n$ denotes the node number in the $x$-direction and $m$ that in the $z$-direction. The equation relating the vector of pressures at each node, $\mathbf{p}$, to that of the external excitation, $\mathbf{q}$, can then be written in the matrix form,

$$
\mathbf{A p}=\mathbf{q}, \quad \text { so that } \quad \mathbf{p}=\mathbf{A}^{-1} \mathbf{q},
$$

and hence used to solve for the pressure distribution in the cochlea. A is a tri-diagonal, block matrix made up of $M \times M$ sub-matrices. The outer diagonal sub-matrices are simple negative identity matrices with zero elements at the locations denoting the cochlear walls and BM, allowing the implementation of the boundary conditions. The centre sub-matrices are themselves tri-diagonal and contain elements defining the boundary conditions of the cochlear walls and BM. The first of these sub-matrices differs as it contains the boundary conditions of the cochlear windows. This large matrix equation was built and solved in MATLAB for the fluid pressure at each node of the model. From these pressures the $\mathrm{BM}$ displacement was determined for both $\mathrm{BC}$ pathways.

\section{Exciting the Model}

The model is excited separately by two BC pathways, the middle ear inertia and the cochlear fluid inertia. The boundary conditions of the walls and windows differ for these two different excitation mechanisms. When the fluid inertia component of $\mathrm{BC}$ is modelled the cochlea is excited by vibrating the walls either side of the $\mathrm{BM}$. The acceleration of the both walls are equal, in the same $z$-direction and given by, $a_{w}=-i \omega v_{w}$. The equation describing the cochlear wall boundary condition for this mechanism is Eq. (2), when $a_{w}=a_{z}$ at the cochlear walls parallel to the BM. For this mechanism the acceleration of the cochlear windows, $a_{w i n}$, is unknown. It is related to the admittance experienced by the cochlear fluid at the windows, looking outwards from the cochlea, by $a_{w i n}=i \omega Y_{w} p(0, z)$ where $p(0, z)$ is the calculated fluid pressure at the cochlear window. The admittance at the oval window in a healthy cochlea were modelled using results from Puria [5] where the stiffness, resistance and mass were found to be, $K_{o w}=830 \mathrm{Nm}^{-1}, R_{o w}=0.1 \mathrm{Nm}^{-1} s$ and $M_{o w}=4.5 \times 10^{-6} \mathrm{~kg}$, which represents a single degree of freedom system with a natural frequency of $2160 \mathrm{~Hz}$ and a $Q$ factor of 0.6 . The round window was modelled only to have a stiffness, $K_{r w}$, equal to a $300 \mathrm{Nm}^{-1}$ [4] and hence an admittance of $Y_{r w}=i \omega / K_{r w}$. The cochlear window boundary conditions are described by Eq. (3), when $a_{x}=a_{w i n}$.

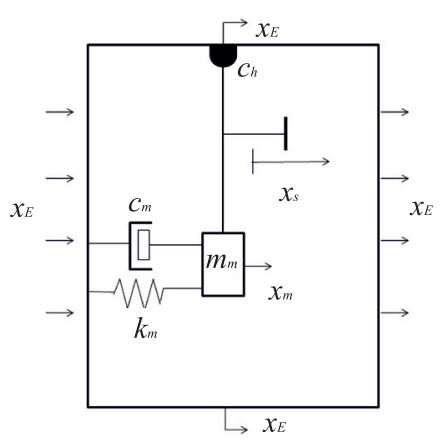

FIGURE 3. Lumped parameter model of the middle ear.
A cochlea diseased by otosclerosis was also modelled, by significantly decreasing the admittance of the oval window, $Y_{o w}$, thus stiffening the window.The acceleration and hence the displacement, at the oval window tended to zero for this condition. The admittance of the round window was unchanged.

The middle ear inertial component stimulates the cochlea at the oval window due to the vibrations of the middle ear. It is assumed that the cochlea is stationary and the walls rigid and hence the pressure gradient at the walls zero. The BM acceleration will only depend on the first term in Eq.6 for a stationary cochlea as the second term will be zero. When the modelling the middle ear inertial component the cochlea model is excited at the oval window by applying an acceleration equal to, $a_{s}=-\omega^{2} x_{s}$, where $x_{s}$ is the frequency dependant stapes displacement, for a given displacement of the skull, $x_{E}$. The ratio, $x_{s} / x_{E}$, is determined by the response of a lumped parameter model of the middle ear ossicles shown in Fig. 3.

The complicated three dimension structure of the ossicles is simply represented by a mass on a lever with an arm, representing the stapes. While, the stiffness and resistance of the tendons and ligaments supporting the ossicles are represented by an angular damped on the hinge, $c_{h}$, and a linear damper, $c_{m}$ and a spring. The box surrounding the system corresponds to the temporal bone, which encases the middle ear cavity in which the ossicles are located. The displacement ratio is equal to,

$$
\frac{x_{s}}{x_{E}}=\frac{1}{L_{r}} \frac{\omega^{2} m_{m}}{k_{m}-\omega^{2} m_{m}+i \omega c_{T}},
$$

where $c_{T}$ is the total damping acting on the system which is equal to $c_{m}+c_{h} / L_{m}$, and is assumed to have a value of $0.12 \mathrm{Nsm}^{-1}$ here. The distance between the hinge and the mass, $L_{m}$, is assumed to be $7 \mathrm{~mm}$ and from the mass to the stapes arm $5 \mathrm{~mm}$ giving a lever ratio, $L_{r}$, of 1.4. The middle ear mass $m_{m}$ assumed to be $m_{m}=5.5 \times 10^{-5} \mathrm{~kg}$ [10] and $k_{m}$ to be $6300 \mathrm{Nm}^{-1}$ giving a natural frequency of $1.7 \mathrm{kHz}$ and a $Q$ value of 5 . 


\section{RESULTS}

For both BC pathways, the cochlear model was excited proportional to a displacement per skull displacement, either at the stapes or cochlea wall. The BM displacement is then calculated as the ratio of BM displacement to external displacement. The BM displacement, relative to the skull displacement, was calculated from the pressure difference across the BM and the BM admittance at each location along the cochlea. The complex BM displacements for both mechanisms, at $1 \mathrm{kHz}$, were calculated and normalised to give the same peak response. The normalised magnitude of the displacement near the base of the cochlea differ for the two methods by about $2 d B$ but the location of the peak response correlates well, Fig. 4A. The phase for both component differ by about 0.25 , cycles but the shape of the phase distribution is the same both mechanisms, Fig. 4B.
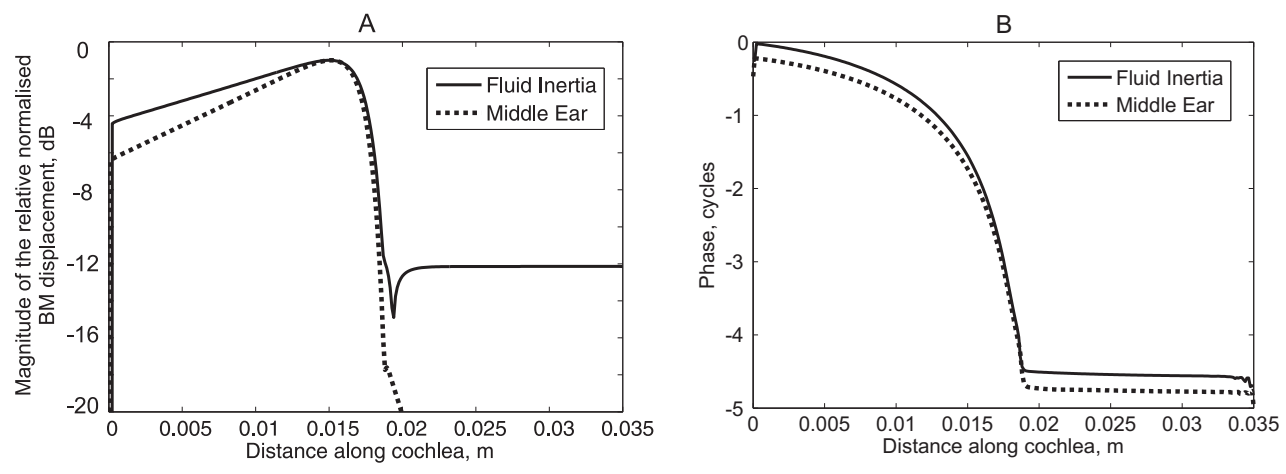

FIGURE 4. Comparison of the magnitude of BM displacements, normalised to give equal peak responses, (A), and the comparison of phase, (B), at $1 \mathrm{kHz}$ for two BC pathways: fluid inertia (solid line) and middle ear inertia (dotted line).

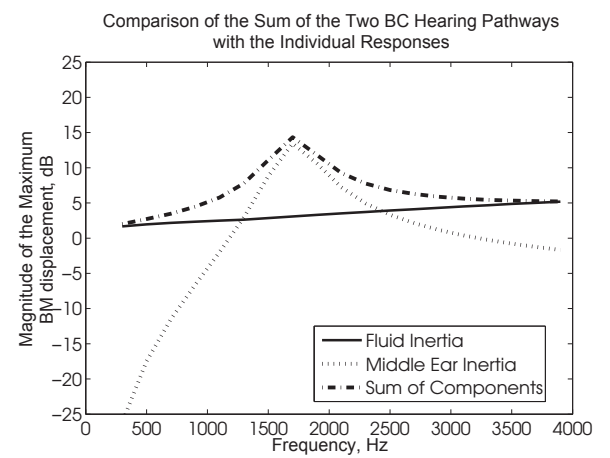

FIGURE 5. Comparison of the maximum basilar membrane response due to the sum of two $\mathrm{BC}$ pathways and the individual responses.
It is the motion of the BM in the cochlea that ultimately causes a hearing sensation and so it is assumed that the magnitude of the maximum BM displacement distribution is directly related to the level of hearing perceived. The maximum BM displacement along the length of the cochlea, for each mechanism, at a specified frequency, is used here to predict the relative significance of the different $\mathrm{BC}$ pathways. The location of the maximum BM displacement varied with frequencies, due the properties of the BM, but, at a given frequency was at the same location for both mechanisms. The total response of the BM is due to the combination of both BC pathways and was found by summing the individual complex BM displacements. It was assumed here that the external displacement driving each mechanism was equal, but, due to the complicated geometry of the real cochlea in reality this may not be true. Fig. 5 shows the magnitude of this maximum displacement for external excitation at different frequencies for the two $\mathrm{BC}$ pathways individually and together.

The model with the parameters assumed here predicts that the total response of the BM, due to the inertia mechanisms of $\mathrm{BC}$ hearing, is dominated by the fluid inertia component below $1.2 \mathrm{kHz}$ and above $2.2 \mathrm{kHz}$. Between these frequencies the middle ear inertia component dominates. The overall $\mathrm{BM}$ response was found to be elevated at $2 \mathrm{kHz}$ by about $12 d B$ as a result of the middle ear inertial component.

\section{Effects of Stiffening the Oval Window}

When otosclerosis is simulated by stiffening the oval window, the stapes becomes motionless and therefore cannot excite the cochlea. This means that under these conditions there will be no contribution to the overall BM response due to the middle ear inertia component and only the fluid inertia component will contribute. The BM response, due to both inertial contributions of $\mathrm{BC}$, with a healthy cochlea was compared to that when the oval window was stiffened, Fig. 6A. It can be seen that there is a reduction $\mathrm{BM}$ response of up to $15 \mathrm{~dB}$ around $2 \mathrm{kHz}$ when the oval window is stiffened. This results agrees with the observed phenomena of patients with otosclerosis having an elevated $\mathrm{BC}$ hearing threshold 
at $2 \mathrm{kHz}$ [1]. The difference in hearing level caused by the stiffening of the oval window was determined by calculating the difference between the BM response with a flexible and stiff oval window. This was then compared against data of patients with otosclerosis and an idealized Carhart notch, Fig. 6B. This model clearly predicts a reduction in hearing at a similar frequency to that found by Carhart [1], and shows a clear "notch" in the region of $2 \mathrm{kHz}$.
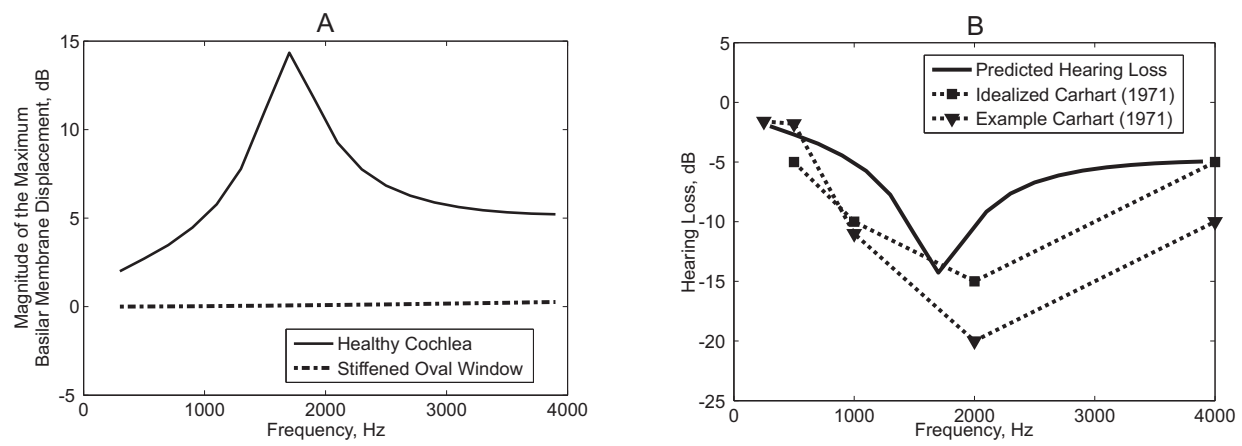

FIGURE 6. (A) Comparison of the magnitude of maximum basilar membrane displacement due to the combination of both BC pathways with and without a stiffened oval window. (B) Comparison of the predicted hearing loss by this model, due to the stiffening of the oval window, an example audiogram from a patient with stapes fixation and an idealised Carhart notch [1].

\section{DISCUSSION}

A 2D finite difference model of the cochlea has been used to investigate the internal response of the cochlea to two forms of inertial excitation that contribute to $\mathrm{BC}$ hearing. It was found that for both mechanisms of exciting the cochlea the distribution of the BM motion along the cochlea was similar, although the overall magnitudes depended differently on frequency. The overall response to the combinations of both mechanisms was calculated and it was found that the fluid inertia component dominated, for the parameters chosen here, below $1.2 \mathrm{kHz}$ and above $2.2 \mathrm{kHz}$ and between these frequencies the middle ear inertia component was dominant. The effects of stiffening the oval window, and hence simulating otosclerosis, were also investigated. It was found that the reduction in hearing predicted by this model was similar to that of patients with otosclerosis [1]. It was assumed that the cochlear fluid is incompressible here, however, this model could be modified to incorporate a compressible fluid and the effects investigated. It could also be used to investigate further near-field coupling due to rocking of the oval window or local excitation of the round window [2].

\section{ACKNOWLEDGEMENTS}

This research is supported by EU project SIFEM (Grants FP7-600933) on the multi-scale modelling and 3D visualization for the inner-ear.

\section{REFERENCES}

[1] Carhart R (1971) Effects of stapes fixation on bone-conduction response. In: Ventry IM, Chaiklin JB, Dixon RF (eds) Hearing Measurement: A book of Readings, New York: Appleton-Century-Crofts, pp. 116-129

[2] Edom E, Obrist D, Henniger R, Kleiser L, Sim JH, Huber AM (2013) The effect of rocking stapes motions on the cochlear fluid flow and on the basilar membrane motion. J Acoust Soc Am 134:3749-3758

[3] Elliott SJ, Shera CA (2012) The cochlea as a smart structure. Smart Mater Struct 21:064001

[4] Gopen Q, Rosowski JJ, Merchant SN (1997) Anatomy of the normal human cochlear aqueduct with functional implications. Hear Res 107:9-22

[5] Puria S (2003) Measurements of human middle ear forward and reverse acoustics: implications for otoacoustic emissions. J Acoust Soc of Am 113:2773-2789

[6] Sohmer H, Freeman S, Geal-Dor M, Adelman C, Savion I (2000) Bone conduction experiments in humans - a fluid pathway from bone to ear. Hear Res 146:81-8

[7] Stenfelt S (2011) Acoustic and physiologic aspects of bone conduction hearing. Adv Otorhinolaryngol 71:10-21

[8] Stenfelt S, Wild T, Hato N, Goode RL (2003) Factors contributing to bone conduction: the outer ear. J Acoust Soc Am 113:902-902

[9] Tonndorf J (1962) Compressional bone conduction in cochlear models. J Acoust Soc Am 34:1127-1131

[10] Wever E, Lawrence M (1954) Physiological Acoustics. Princeton: Princeton University Press 